\title{
Stochastic Binary Sensor Networks for Noisy Environments
}

\author{
Thinh Nguyen \\ School of EECS \\ Oregon State University \\ Corvallis, OR 97331, USA \\ thinhq@eecs.oregonstate.edu
}

\author{
Dong Nguyen \\ School of EECS \\ Oregon State University \\ Corvallis, OR 97331, USA \\ nguyendo@eecs.oregonstate.edu
}

\author{
Duc Tran \\ Computer Science Department \\ University of Dayton \\ Dayton, OH 45469, USA \\ duc.tran@notes.udayton.edu
}

\begin{abstract}
This paper proposes a stochastic framework for detecting anomalies or gathering interesting events in a noisy environment using a sensor network consisting of binary sensors. A binary sensor is an extremely coarse sensor, capable of measuring data to only 1-bit accuracy. Our proposed stochastic framework employs a large number of cheap binary sensors operating in a noisy environment, yet collaboratively they are able to obtain accurate measurements. The main contributions of this paper are: (a) The theoretical accuracy analysis of the proposed stochastic binary sensor network, (b) an adaptive data collection framework based on the current measurements in order to reduce the energy consumption, and (c) a novel coding scheme for energy-efficient routing. To quantify the performance of our proposed stochastic approach, we present the simulation results of two stochastic binary sensor networks for anomaly detection using our proposed coding scheme and adaptive data gathering framework. For many scenarios, our proposed framework can reduce the energy consumption over the traditional approach by an order of magnitude.
\end{abstract}

\section{INTRODUCTION}

In recent years, sensor networks have emerged as an important class of networks for many military and commercial applications [1][2][3]. A sensor network is a collection of wireless communication nodes. Each node is capable of sensing the environment and communicating the measured data to the neighboring nodes, and eventually to the external users. The majority of sensor networks are designed to collect data [4][5] or to perform anomaly detection [6][7] while achieve the goal of high accuracy and minimum energy consumption.

Accuracy. A special and important situation arises when all the sensors measure the same underlying signal $x$. However due to the environmental noise, each sensor $i$ measures a different value $x_{i}=x+n_{i}$ where $n_{i}$ is an additive noise sample. In this scenario, the objective of a sensor network is to accurately determine the underlying value $x$ through collaborations among the sensors. For example, the nodes in a sensor network for anomaly or intrusion detection often exchange data among each other to increase the detection accuracy. In a real-world scenario, these sensors can be the magnetic sensors in the ocean whose tasks are to determine the magnetic signature of a submarine. This collaboration of nodes for the accurate estimation of data in the sensor networks is one of the main contributions of our paper.
Energy Efficiency. Since a sensor is typically a batteryoperated device, minimizing energy consumption should also be considered. If the measured data among the nodes are spatially correlated, a node can jointly compress its data and its neighbor's data in order decrease the transmission energy [8][9]. The higher correlation of data measured among the sensors results in more energy saving. In our proposed stochastic binary sensor network, the underlying measured data at each sensor are assumed to be identical or highly correlated. This high correlation enables us to employ a novel coding scheme to reduce the energy consumption. The energy saving is further reduced by using our adaptive data gathering framework.

In this paper, we present a stochastic binary sensor network that (a) achieves good accuracy, (b) enables the graceful degradation of data quality in the presence of sensor's failures, and (c) reduces the energy due to the adaptive data collection technique and a novel coding scheme. In the proposed sensor network, each sensor employs only 1 bit of information per measurement, but collectively, accurate data measurements can be obtained. By allowing only 1-bit measurement, the sensors can be made at low cost and, therefore, are easy to replace or to discard. Furthermore, the higher number of working sensors results in higher data quality. In this paper, we assume that the data from the working sensors can be routed to the processing node. Thus, the data quality depends only on the number of working sensors.

Our paper is organized as follows. In Section II, we discuss the assumptions and objectives of the stochastic binary sensor network. Section III is devoted to the theoretical accuracy analysis of the stochastic binary sensor network in a variety of environments. In Section IV, we present a novel coding scheme to be used for energy-efficient routing. Section V provides the simulation results for two binary sensor networks that employ the adaptive data collection technique and the coding scheme to reduce energy consumption. Finally, we provide a few concluding remarks in Section VI.

\section{Model for the Stochastic Binary Sensor NETWORK}

In this section, we discuss the mathematical model of data estimation in a stochastic binary sensor network. For the 
purpose of illustration, we begin with a simple scenario in which four nodes in a sensor network are aligned in a straight line as shown in Figure 1. We assume that the data $x(t)$ at time $t$ is identical (or highly correlated) within the coverage of the sensors. In other words, the data may vary temporally but not spatially. Each node $i$ is to measure the underlying data $x(t)$ at time $t$. Theoretically, without noise, all the nodes would obtain identical measurements at time $t$. However, in non-ideal situations where either internal or external noise is present, an accurate measurement cannot be obtained using only a single node. Another argument against using a single accurate sensor is the lack of robustness since a single failure would stop the entire data collection process.

Based on the discussion above, our model consists of many sensors. Each sensor measures its data at time $t$ as $x_{i}(t)=x(t)+n_{i}(t)$ where $n_{i}(t)$ is independent and identically distributed noise at node $i$. The measurements from different nodes are forwarded to a processing node whose task is to accurately determine the value $x(t)$. For example, if node 4 is chosen to be the processing node, then such node is responsible for determining the value of $x(t)$ based on all the measurements it receives from nodes 1, 2, and 3. Figure 1 shows how measurements are forwarded from nodes 1,2 , and 3 to node 4 .

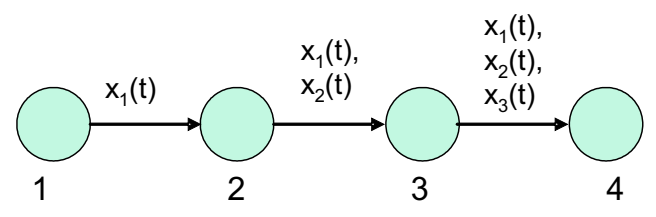

Fig. 1. A simple sensor network. Measured data are relayed to node 4 .

The classical method for estimating value of $x(t)$ [10] is given by $\hat{x}(t)=\frac{1}{N} \sum_{i=1}^{N} x_{i}(t)$. where $N$ is the number of sensors. The larger the value of $N$, the higher the accuracy. This method assumes an accurate representation of $x_{i}(t)$. Theoretically, if we have an infinite precision representation of $x_{i}(t)$, then the mean estimated error $\sqrt{E\left[(x-\hat{x})^{2}\right]}$ using the classical method would decrease by a factor of $\frac{1}{\sqrt{N}}$. However, an accurate representation of $x_{i}(t)$ implies that each sensor must be able to resolve a small difference in the measured data. For example, when measuring the temperature, a sensor node must be able translate temperatures to electrical signals in a fairly precise manner. For example, $68.2 \mathrm{~F}$ and $68.5 \mathrm{~F}$ would result in two electrical signals of 0.90 volts and 0.92 volts, respectively. In addition, an accurate representation $x_{i}(t)$ requires a larger number of bits, hence more bits must be transmitted per measurement per sensor.

Based on the discussion above, our proposed framework eliminates the need for high resolution sensors. In particular, each sensor only makes a decision whether its measured data is greater or smaller than a certain threshold. This simplification allows one to build a sensor network consisting of cheap and simple sensors. In particular, we make the following assumptions:
1) Signal $x(t)$ is a random process with zero mean. The zero-mean assumption is for ease of analysis.

2) Additive noise $n_{i}(t)$ is independent and identically distributed around zero. This assumption holds for geographically far-apart nodes which are likely to have uncorrelated environmental noise samples.

3) Each sensor node is only able to detect the sign of $x_{i}(t)$. This mean the the threshold value is equal to 0 .

Accuracy. Based on the above assumptions, our goal is to estimate $x(t)$ as $\hat{x}(t)=f\left(y_{1}(t), \ldots, y_{N}(t)\right)$ for some function $f($.$) , where y_{i}(t)=\operatorname{sgn}\left(x_{i}(t)\right)$, given the statistics of $x(t)$ and of the noise $n_{i}(t)$. We do not make any assumption on temporal correlation of the data, i.e., $x(t-a)$ cannot be used to estimate $x(t)$. Thus, we shall omit the index $t$ in all the variables, e.g., $x_{i}(t)$ will become $x_{i}$.

There are two important performance indicators for our sensor network: the Mean Square Error $(M S E)$ and the Conditional Mean Square Error (CMSE) which are defined as

$$
\begin{aligned}
M S E & \triangleq E\left[\left(x-f\left(y_{1}, \ldots, y_{N}\right)\right)^{2}\right], \\
C M S E & \triangleq E\left[\left(x-f\left(y_{1}, \ldots, y_{m}\right)\right)^{2} \mid y_{1}, \ldots, y_{m}\right],
\end{aligned}
$$

where $m \leq N$. The $M S E$ characterizes the measured data quality of a sensor network, while $C M S E$ characterizes the average amount of errors given a set of observations. Thus, the $M S E$ enables us to characterize the performance of our sensor network in a certain environment. On the other hand, the $C M S E$ is useful for adaptive data gathering to reduce the energy consumption.

Adaptive Data Gathering. To illustrate the energy reduction based on adaptive processing, we consider the following example. Suppose that a sensor network consisting of $N$ nodes is designed to continuously measure a signal $x$ and alarms the population whenever it detects $x>a$. In a real world scenario, $x>a$ may represent an anomaly such as the presence of an intruder or a dangerous chemical agent. Using all $N$ data points, the processing node can estimate $x$ as $f\left(y_{1}, y_{2}, \ldots, y_{N}\right)$ and determine whether $f\left(y_{1}, y_{2}, \ldots, y_{N}\right)>a$. Since $f\left(y_{1}, y_{2}, \ldots, y_{N}\right)$ is an estimation of $x$, it is also useful determine the confidence level of this estimation in order to reduce the number of false alarms or to avoid missing an anomaly. As will be shown shortly, the accuracy of the estimation depends on the current estimated value of $x$ and on the number of data samples. Thus, if after a number of transmissions $n<N$, the intermediate nodes can estimate that $x<a$, i.e., no anomaly, with high confidence, then subsequent transmissions will be not be necessary, resulting in an overall energy reduction of the network.

Coding. Energy consumption can be further reduced by employing our coding scheme which exploits the network topology. The main idea is that, to estimate the signal, the processing node only needs to know the summary information such as the number of 1's. Thus, each node can reduce the number of transmitted bits accordingly based on its positions. We discuss this in detail in Section IV. We now begin with 
the accuracy analysis.

\section{ACCURACY ANALYSIS}

In this section, we analyze the accuracy of the $M S E$ and $C M S E$ for the proposed binary stochastic sensor network operating in a typical environment where the signal and the additive noise are both uniformly distributed ${ }^{1}$. We compute $M S E \triangleq E\left[(x-\hat{x})^{2}\right]$ and $C M S E \triangleq E\left[(x-\hat{x})^{2} \mid \hat{x}\right]$.

Given a signal $x$ and the observations at different nodes $y_{1}, y_{2}, \ldots, y_{N}$, we want to determine a good estimator $\hat{x}=$ $f\left(y_{1}, y_{2}, \ldots, y_{N}\right)$ for $x$, where $y_{i}=\operatorname{sgn}\left(x+n_{i}\right)$. When the signal and noise distributions are uniformly distributed over $[-\alpha, \alpha]$ and $[-\beta, \beta]$, respectively, and $\beta \geq \alpha$, a good estimator is

$$
\hat{x}=\frac{\beta}{N} \sum_{i=1}^{N} y_{i}
$$

We note that, although $x \in[-\alpha, \alpha]$, the estimator $\hat{x}=$ $\frac{\alpha}{N} \sum_{i=1}^{N} y_{i}$ is actually less accurate than $\hat{x}=\frac{\beta}{N} \sum_{i=1}^{N} y_{i}$ in terms of $M S E$. We prove the following theorem regarding the accuracy of our stochastic binary sensor network.

Theorem 3.1: If the signal and the additive noise are uniformly distributed in the intervals $[-\alpha, \alpha]$ and $[-\beta, \beta]$, respectively, with $\beta \geq \alpha$, then using the estimator in Equation (3) with $N$ sensors, it is

$$
\begin{array}{r}
M S E=\frac{3 \beta^{2}-\alpha^{2}}{3 N}, \\
C M S E=\frac{A}{B},
\end{array}
$$

where

$$
\begin{array}{r}
A=\sum_{k=0}^{j} \sum_{i=0}^{N-j} \frac{(-1)^{i}}{\beta^{i+k}}\left(\begin{array}{l}
j \\
k
\end{array}\right)\left(\begin{array}{c}
N-j \\
i
\end{array}\right) \alpha^{i+k+1} \\
\left(\alpha^{2} \frac{1+(-1)^{i+k}}{i+k+3}+2 \beta \alpha\left(\frac{2 j}{N}-1\right) \frac{(-1)^{i+k}-1}{i+k+2}\right. \\
\left.+\beta^{2}\left(\frac{2 j}{N}-1\right)^{2} \frac{1+(-1)^{i+k}}{i+k+1}\right)
\end{array}
$$

and

$$
B=\sum_{k=0}^{j} \sum_{i=0}^{N-j} \frac{(-1)^{i}}{\beta^{i+k}}\left(\begin{array}{l}
j \\
k
\end{array}\right)\left(\begin{array}{c}
N-j \\
i
\end{array}\right) \frac{1+(-1)^{i+k}}{i+k+1} \alpha^{i+k+1}
$$

Proof (outline): Let $f_{n}(x)$ and $f(x)$ be the probability density functions of the noise and of the signal, respectively. Also denote the number of non-negative samples as $j$, then $\hat{x}=\beta\left(\frac{2 j}{N}-1\right)$. Now,

$$
\begin{aligned}
E\left[(x-\hat{x})^{2} \mid x\right]= & x^{2}-2 x \beta E\left[\left(\frac{2 j}{N}-1\right) \mid x\right] \\
& +\beta^{2} E\left[\left(\frac{2 j}{N}-1\right)^{2} \mid x\right]
\end{aligned}
$$

\footnotetext{
${ }^{1} \mathrm{We}$ also have results for the case that the signal and the additive noise are Gaussian distributions, but because of the limit of the paper, we do not illustrate it here
}

Given $x, j$ is a binomial random variable, hence

$$
\begin{aligned}
E[j \mid x] & =N q \\
E\left[j^{2} \mid x\right] & =(N q)^{2}+N q(1-q),
\end{aligned}
$$

where $q$ is the probability that $\operatorname{sgn}\left(x+n_{i}\right)>0$,

$$
q=\frac{1}{2}\left(\frac{x}{\beta}+1\right) \text {. }
$$

Now, substituting $E[j \mid x]$ and $E\left[j^{2} \mid x\right]$ into Equation (8), we obtain

$$
E\left[(x-\hat{x})^{2} \mid x\right]=\frac{\beta^{2}-x^{2}}{N} .
$$

Next, the mean square error is

$$
\begin{aligned}
M S E=E\left[(x-\hat{x})^{2}\right] & =\int_{-\infty}^{\infty} E\left[(x-\hat{x})^{2} \mid x\right] f(x) d x \\
& =\int_{-\alpha}^{\alpha} \frac{\beta^{2}-x^{2}}{N} \frac{1}{2 \alpha} d x \\
& =\frac{3 \beta^{2}-\alpha^{2}}{3 N}
\end{aligned}
$$

To obtain $C M S E$, let $f(x \mid j)$ be the conditional density function of $x$ given $j$-the number of non-negative samples, we have:

$$
\begin{aligned}
E\left[(x-\hat{x})^{2} \mid \hat{x}\right] & =E\left[(x-\hat{x})^{2} \mid j\right] \\
& =\int_{-\infty}^{\infty}(x-\hat{x})^{2} f(x \mid j) d x \\
& =\frac{\int_{-\alpha}^{\alpha}(x-\hat{x})^{2} q^{j}(1-q)^{N-j} d x}{\int_{-\alpha}^{\alpha} q^{j}(1-q)^{N-j} d x}
\end{aligned}
$$

Replacing $q$ in Equation (13), we have

$$
E\left[(x-\hat{x})^{2} \mid j\right]=\frac{\int_{-\alpha}^{\alpha}(x-\hat{x})^{2}\left(1+\frac{x}{\beta}\right)^{j}\left(1-\frac{x}{\beta}\right)^{N-j} d x}{\int_{-\alpha}^{\alpha}\left(1+\frac{x}{\beta}\right)^{j}\left(1-\frac{x}{\beta}\right)^{N-j} d x}
$$

Now, letting $A=\int_{-\alpha}^{\alpha}(x-\hat{x})^{2}\left(1+\frac{x}{\beta}\right)^{j}\left(1-\frac{x}{\beta}\right)^{N-j} d x$ and $B=\int_{-\alpha}^{\alpha}\left(1+\frac{x}{\beta}\right)^{j}\left(1-\frac{x}{\beta}\right)^{N-j} d x$, then $E\left[(x-\hat{x})^{2} \mid j\right]=\frac{A}{B}$, Using binomial expansion, we obtain $A$ and $B$ in Equation (6) and Equation (7) respectively. Q.E.D.

Theorem 3.1 states that the $M S E$ is inversely proportional to $N$. This agrees with our intuition that a larger $N$ leads to a smaller estimation error. Theorem 3.1 also implies that the $M S E$ is minimized when $\beta=\alpha$, or when the signal and noise have identical statistics. As seen in Figure 2(a), the $M S E$ curve for the noise in the range $[-1,1]$ is minimal. Furthermore, the $M S E$ becomes larger as the gap between the noise range and the signal range increases. One important observation is that the $M S E$ asymptotically vanishes as the number of sensors $N$ increases. This is important since we can guarantee an arbitrarily small estimation error, even in an environment with an arbitrarily large noise when using an appropriately large number of nodes. Figure 2(a) shows this asymptotical decrease of $M S E$ as the number of nodes increases. 


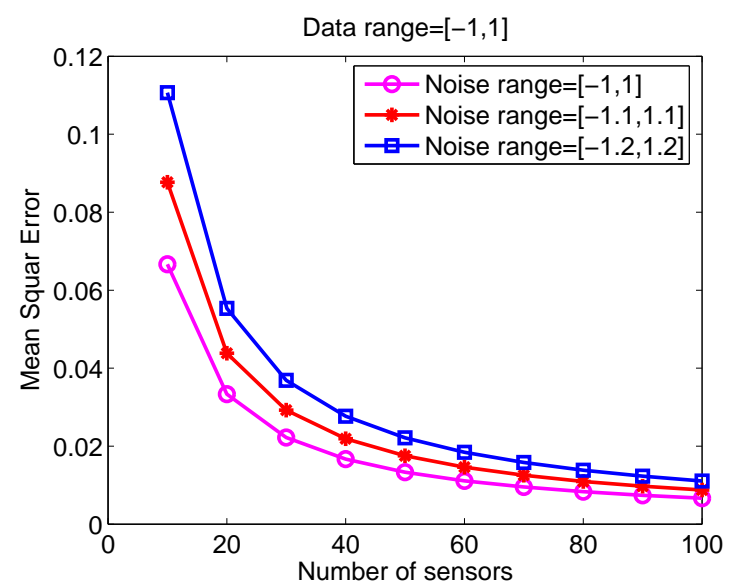

(a)

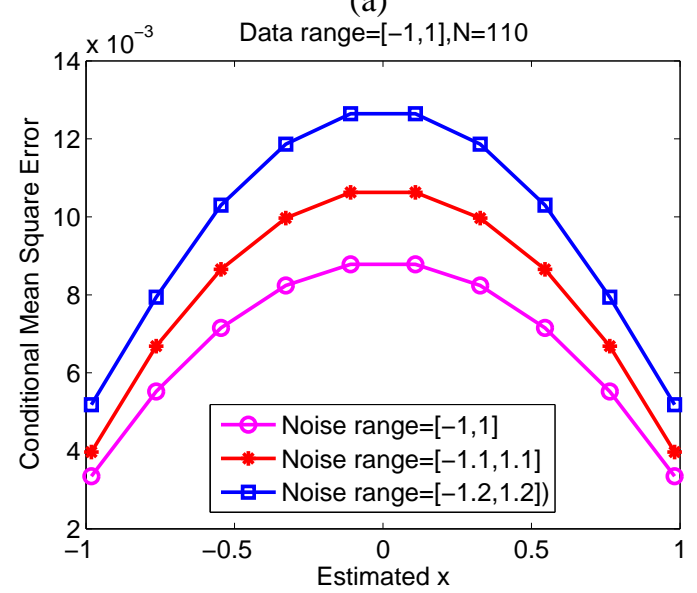

(b)

Fig. 2. Performance of a binary sensor network with uniform signal over $[-1,1]$ under the different uniform noises. (a) MSE as a function of number of sensors; (b) CMSE as a function of $\hat{x}$, with the number of nodes $N=$ 110.

Computing the $C M S E$ is rather complicated; however, it can be computed once for all the possible values of $\hat{x}$ and the results are stored in a table at each sensor. When the adaptive data collection technique is used, each sensor can determine the corresponding $C M S E$ given $\hat{x}$ using the look-up table. We note that the $C M S E$ depends on $\hat{x}\left(\hat{x}=\beta\left(\frac{2 j}{N}-1\right)\right)$ and $N$. In particular, for a uniform signal and a uniform noise, the $C M S E$ increases when the magnitude of $\hat{x}$ decreases, and vice versa as shown in Figure 2(b). Thus, an intermediate node can compute $\hat{x}$ after collecting a number of samples from other nodes and based on the value of $\hat{x}$ and the corresponding estimation error, it decides whether or not to continue relaying the data to next node in order to save energy. This decision is application dependent as will be discussed in Section V.

\section{ENERgy EFFICIENT CODING}

In this section, we present the energy efficient coding. Most often, the energy saving is obtained through efficient routing. However, we do not address the routing issue in this paper. Instead, we assume that the route for gathering data is already established. Our objective is to further improve energy efficiency through coding. We assume that the energy consumption by the sensor nodes is proportional to the number of transmitted bits. Therefore, our objective is to minimize the number of bits sent in the network. To illustrate our approach, we consider two topologies of networks: straight line and tree.

Consider four sensor nodes arranged in a straight line as shown in Figure 1. In our stochastic sensor network, a node sends one bit per its measured sample $x(t)$, and depending on its position, it also relays many bits from other nodes. Thus, without coding, the total number of bits to send in this simple network is $1+2+3=6$ bits.

However, the processing node 4 only needs to know the number of non-negative samples. In other words, to estimate $x$, node 4 does not need to know whether the measured values at each node is -1 or 1 . Thus, instead of sending all 6 bits, node 3 may need to send to node 4 only 2 bits (4 possible patterns) to represent whether the number of non-negative samples is $0,1,2$, or 3. Given this topology, it is impossible for node 4 to receive a number of non-negative samples greater than 4. Similarly, node 2 needs to send only 2 bits to node 3 to represent whether the number of non-negative samples that it has received so far is 0,1 , or 2 .

While the example above shows a modest energy reduction, for a sensor network consisting of a large number of nodes arranged in a straight line, this coding technique can result in substantial energy reduction. We prove the following theorem.

Theorem 4.1: Given $N$ sensor nodes arranged in a straight line with the processing node at one end, then the maximum number of bits which need to be sent per sample in the stochastic binary sensor network with coding is

$$
B_{1}=N(m+1)+1-2^{m+1},
$$

and without coding is

$$
B_{2}=\frac{N(N-1)}{2}
$$

where $m=\left\lfloor\log _{2} N\right\rfloor$.

Proof: Assume that node $N$ is the processing node. Without loss of generality, we have $2^{m} \leq N<2^{m+1}$, for $m=$ $\left\lfloor\log _{2} N\right\rfloor$. Then, the total number of bits sent by nodes 1 to $2^{m}-1$ is

$$
A_{1}=\sum_{i=1}^{m} i 2^{i-1}
$$

We can easily find out

$$
A_{1}=(m+1) 2^{m}-2^{m+1}+1 .
$$

Also, the total number of bits sent by the remaining nodes is

$$
A_{2}=\left(N-2^{m}\right)(m+1) \text {. }
$$

Adding $A_{1}$ and $A_{2}$, we obtain $B_{1}$. $B_{2}$ is easily obtained using arithmetic sum. Q.E.D.

For the tree topology with $k$ branches, we prove the following theorem for data gathering. Data gathering starts from the nodes in the lowest to highest level. 


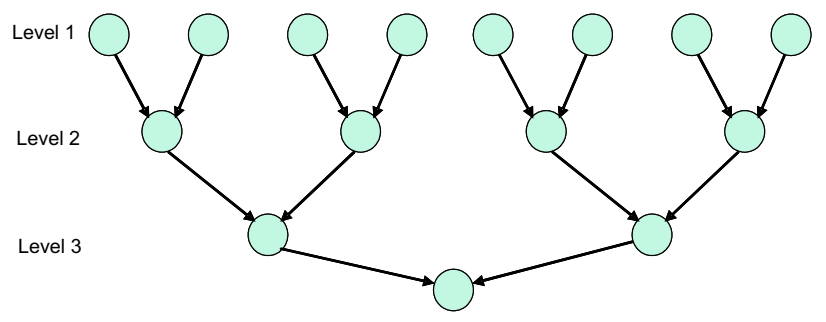

Fig. 3. Tree topology for data gathering.

Theorem 4.2: Using coding, the maximum number of transmitted bits per sample in a tree topology with $k$ branches is

$$
B_{k}=k^{m-1}+\sum_{i=2}^{m-1}\left\lceil\log _{2} \frac{k^{i}-1}{k-1}\right\rceil k^{m-i},
$$

where $m$ is the number of levels. For $k=2$,

$$
B_{2}=2\left(2^{m}-m-1\right) \text {. }
$$

Proof: For an $m$-level tree, there are $k^{m-1}$ leaf nodes and each leaf node sends 1 bit of data. Therefore, the total number of bits sent by the leaf nodes is $k^{m-1}$ bits. Now, each internal node needs to relay data for all its predecessors. If a node is at level $i>1$, it has $\frac{k^{i}-1}{k-1}-1$ predecessors (using the geometric sum). Since node $i$ also needs to send 1 bit of its measured data, the maximum total number of coded bits sent by node $i$ is $\left[\log _{2} \frac{k^{i}-1}{k-1}\right]$. Finally, there are $k^{m-i}$ nodes at level $i$, hence the total number of coded bits sent by all the nodes (excluding the processing node at level $m$ ) is

$$
B_{k}=k^{m-1}+\sum_{i=2}^{m-1}\left\lceil\log _{2} \frac{k^{i}-1}{k-1}\right\rceil k^{m-i} .
$$

For $k=2$, we have

$$
B_{2}=2^{m} \sum_{i=1}^{m-1} i 2^{-i}
$$

We can prove that

$$
\sum_{i=1}^{m-1} i 2^{-i}=2\left[1-\frac{m+1}{2^{m}}\right]
$$

Finally, $B_{2}=2^{m} \sum_{i=1}^{m-1} i 2^{-i}=2\left(2^{m}-m-1\right)$. Q.E.D.

\section{Simulation Results For Binary SEnsor NETWORKS}

In this section, we characterize the trade-off between the energy consumption and the accuracy of the stochastic binary sensor networks for detecting anomalies through simulations. In particular, we consider two special topologies for simulations. The first topology is a straight-line topology consisting of 128 binary sensor nodes arranged in a straight line, with the processing node at one end. The measured data flow from one end of the line to the processing node at the other end. Data is accumulated along the way so that the processing node have all the measured data. The second topology is a tree topology consisting of 127 binary sensor nodes. Data are relayed from the leaf nodes to the internal nodes, and subsequently to the processing node as shown in Figure 3.

The main idea for reducing energy consumption in these networks is for a node to stop relaying data to the processing node if it determines with high confidence that the current estimated data is not anomalous. In particular, in these simulations, we consider a data point $x$ anomalous if the estimated $a b s(\hat{x}) \geq a$ and $C M S E=E\left[(x-\hat{x})^{2} \mid \hat{x}\right]<b$ where $a$ and $b$ are some threshold values set by the applications. Using this framework, each node would estimate the current data based on its own measurement and the measurements relayed to it from other nodes. We note that the CMSE is employed in the decision making of a node to express the confidence level in the estimated data $\hat{x}$.

Using this model, a node in both straight-line and tree sensor networks operates as follows.

1) Initially, if a node is a leaf node in a tree topology or the first node in a line topology, it would send its data to the next node.

2) An internal node may send data only if it receives data from at least one node. This implies that, if all the predecessor nodes of a node determine that no further transmission is necessary, that node will honor the predecessor's decision.

3) If a node receives data from its predecessor node(s), it estimates the current value $\hat{x}$ and the $C M S E$ based on its own measurement and the relayed measurements from other node(s). If $a b s(\hat{x})<a$ and $C M S E<b$, it stops relaying data to the next node. Otherwise, it sends data to the next node.

To characterize the energy reduction due to our proposed coding technique and the adaptive data collection, for each topology, we perform the simulations for sensor network with coding and without coding. The threshold value $a$ is set to 0.5 , while $b$ is varied to characterize the trade-off between energy consumption and accuracy. Figure 4 shows the number of transmitted bits per data measurement as a function of $C M S E$ for the straight-line topology. It can be noticed that, using coding reduces the number of transmitted bits approximately by a factor of 10 compared to without using coding. Also, if an application allows a larger estimation error, further energy reduction can be obtained, e.g., the number of transmitted bits with the $C M S E=0.18$ is 8 times smaller than that of using the $C M S E=0.01$. Similarly, Figure 5 shows substantial saving of using coding in the tree topology. On the other hand, the adaptive data collection technique does not reduce the energy consumption as much. We note that the tree topology is much more energy efficient that the straight-line topology. This is because a transmitted bit in a tree topology does not have to be relayed many times as in the line topology. However, the coverage of a straight-line topology is larger than that of a tree topology. At the limit, if all the nodes reside on one chip, then the energy would be minimum since no off-chip transmission is necessary. 


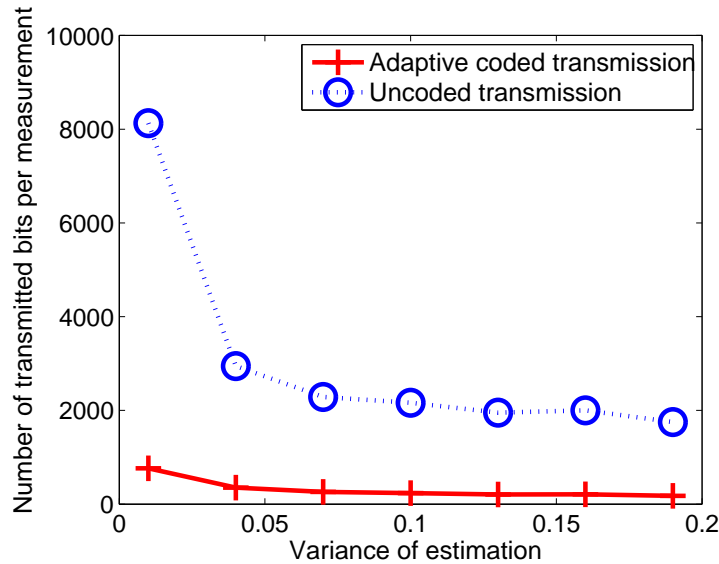

Fig. 4. Number of transmitted bits per measurement as a function of $C M S E$ for a straight line topology.

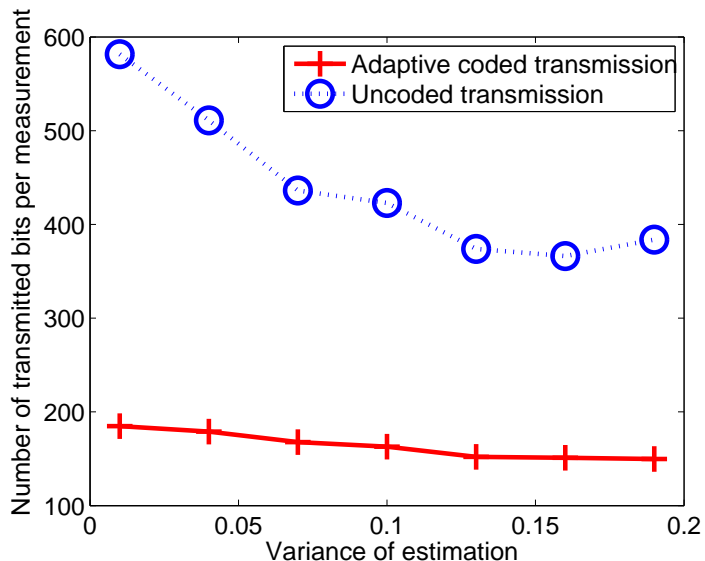

Fig. 5. Number of transmitted bits per measurement as a function of $C M S E$ for a tree topology.

\section{CONCLUSIONS}

In summary, we have proposed a stochastic framework for detecting anomalies or gathering interesting events in a noisy environment using a sensor network consisting a large number of cheap binary sensors operating in a noisy environment. We present the theoretical analysis of the accuracy of such sensor networks in different environments. We also propose an adaptive data collection framework based on the current measurements and a novel coding scheme in order to reduce the energy consumption. The simulation results of two stochastic binary sensor networks for anomaly detection using our proposed coding scheme and adaptive data gathering show that energy consumption can be reduced substantially, e.g., a factor of 10 for many scenarios.

\section{REFERENCES}

[1] I Akyildiz, W. Su, Y. Sankarasubramaniam, and E. Cayirci, "A survey on sensor networks," IEEE Communications Magazine, 2002.

[2] J. Kahn, R. Katz, and K. Pister, "Mobile networking for smart dust," in ACM/IEEE International Conference on Mobile Computing and Networking, august 199.
[3] J. Rabaey, J. Ammer, J. Silva, and D. Patel, "Picoradio:ad-hoc wireless networking of ubquituous low-energy sensor/monitor nodes," in Proceedings of the IEEE Computer Society Annual Workshop on VLSI, april 2000.

[4] J. Heidemann, F. Silva, C. Intanagonwiwat, R. Govindan, D. Estrin, and D. Ganesan, "Building efficient wireless sensor networks with low-level naming," in 18th ACM Symposium on Operating Systems Principles, october 2001.

[5] C. Intanagonwiwat and R. Govindan D. Estrin, "Directed difussion: A scalable and robust communication paradigm for sensor networks," in ACM/IEEE International Conference on Mobile Computing and Networks, august 2000.

[6] W. Du, L. Fang, and P. Ning, "Lad: Localization anomaly detection for wireless sensor networks," in In The 19th International Parallel and Distributed Processing Symposium (IPDPS), April 2005.

[7] T. Vercauteren, D. Guo, and X. Wang, "Joint multiple target tracking and classification in collaborative sensor networks," IEEE Journal On Selected Areas in Communications, vol. 23, pp. 714-723, 2005.

[8] R. Cristescu, B. Beferull-Lozano, and M. Vetterli, "On network correlated data gathering," in Proceeding of INFOCOM, 2004.

[9] S. Lindsey, C. Ragavendra, and K. Sivalingam, "Data gathering algorithms in sensor networks using energy metrics," IEEE Transactions on Parallel and Distributed Systems, vol. 23, pp. 776-787, 2002.

[10] M. Hayes, Statistical Digital Signal Processing and Modeling, Wiley, 1996. 